Revista científica, arbitrada e indizada, bajo la modalidad electrónica.

Recibido: 08/01/2021

Aceptado: 19/01/2021

\title{
Trata de personas para el aprovechamiento sexual en Venezuela
}

Trafficking in persons for sexual use in Venezuela

\author{
Rainier José Rivero Colina \\ Universidad Yacambú- Venezuela \\ rainierjriveroc@gmail.com \\ Joel José Piña \\ Universidad Yacambú- Venezuela \\ Jotasilver87@gmail.com
}

\section{RESUMEN}

Este trabajo tiene como propósito analizar la trata de personas para el aprovechamiento sexual en Venezuela. La investigación se encuentra inmersa en un enfoque postpositivista, siguiendo los lineamientos del paradigma cualitativo, de igual manera, es de tipo documental sociojurídico, con un diseño bibliográfico. Enfatizando su preminencia con aportes de un paradigma interpretativo y multi-métodos. El universo documental se encuentra conformado por 47 documentos. El trabajo desarrollado da inicio con la elección del tema, realizando un acopio bibliográfico, seguidamente, los datos recopilados de los referentes teóricos son registrados en fichas hemerográficas y bibliográficas, para tenerlos disponibles y localizarlos de manera oportuna. Luego son sistematizados todos los datos, a los fines de efectuar el análisis de contenido. En este sentido, los hallazgos dejan en evidencia que la situación socioeconómica ha llevado a una cantidad bastante considerable de personas, a salir del país por diferentes vías y a diversos países, este hecho apalanca el fortalecimiento de la actividad delictiva, porque los traficantes aprovechan ese escenario para penetrar con sus estrategias manipuladoras, aquellos sectores con mayor vulnerabilidad. De igual forma, es indudable que el aprovechamiento sexual, producto de la trata de personas se ha infiltrado en la sociedad venezolana, a todos los niveles, deteriorando más aun la calidad de vida de la población. También es evidente que el país ha venido fortaleciendo su marco legal para luchar contra la trata de personas, aun cuando todavía no se tiene un instrumento en específico, que agrupe y regule de manera particular la actividad delictiva. Sin embargo, todas las acciones ejecutadas no han logrado la meta de erradicar o al menos minimizar este terrible flagelo.

Palabras Clave: Trata de personas, Aprovechamiento sexual, Vulnerabilidad social. 


\section{Revista científica, arbitrada e indizada, bajo la modalidad electrónica.}

\section{ABSTRACT}

The purpose of this work is to analyze human trafficking for sexual exploitation in Venezuela. The research is immersed in a postpositivist approach, following the guidelines of the qualitative paradigm, in the same way; it is of a socio-legal documentary type, with a bibliographic design. Emphasizing its preminence with contributions from an interpretive and multi-method paradigm. The documentary universe is made up of 47 documents. The work developed begins with the choice of the topic, carrying out a bibliographic collection, and then the data collected from the theoretical references are recorded in hemerographic and bibliographic files, to have them available and locate them in a timely manner. Then all the data are systematized, in order to carry out the content analysis. In this sense, the findings make it clear that the socioeconomic situation has led a considerable number of people to leave the country through different routes and to various countries, this fact leverages the strengthening of criminal activity, because traffickers take advantage of this scenario to penetrate with their manipulative strategies, those sectors with the greatest vulnerability. Similarly, there is no doubt that sexual exploitation, a product of human trafficking, has infiltrated Venezuelan society at all levels, further deteriorating the quality of life of the population. It is also evident that the country has been strengthening its legal framework to combat human trafficking, even though it still does not have a specific instrument that groups and regulates criminal activity in a particular way. However, all the actions carried out have not achieved the goal of eradicating or at least minimizing this terrible scourge.

Keywords: Human trafficking, Sexual exploitation, Social vulnerability

\section{INTRODUCCIÓN}

El Protocolo de Palermo instituye la presencia de diferentes etapas del delito de trata de personas, entre las cuales se encuentran la captación, el transporte o traslado, la acogida o recepción y finalmente la explotación, fin último de la trata, que debe permear cada una de las respectivas fases.

De acuerdo con la OMS (2013), la trata de personas:

Ha recibido creciente atención mundial durante el último decenio. Inicialmente, el tráfico de mujeres y niñas para trabajo sexual forzado, y en menor grado para servidumbre doméstica, eran el único foco de las actividades de sensibilización y asistencia. Hoy existe un mayor reconocimiento de que se trafica con mujeres, niños y hombres para explotación sexual y laboral de diversas formas. (p.1).

Con relación a lo planteado, de acuerdo a Chávez et al., (2014), América Latina se destaca por ser una zona de origen, tránsito y destino de trata interna e internacional, en la 


\section{Revista científica, arbitrada e indizada, bajo la modalidad electrónica.}

cual "la trata de personas, principalmente de mujeres y niñas o adolescentes, se centra en la explotación sexual” (p.18).

Como es señalado por los teóricos que fundamentan las fuentes de información utilizadas en este trabajo, esta actividad delictiva moviliza millones de dolares anualmente. De igual manera, afecta los derechos más fundamentales de las víctimas, que incluyen no solo a mujeres mayores de edad, sino también a niños y niñas y en menor cantidad a los hombres. Vinculando sus estrategias manipuladoras, explotando la vulnerabilidad de las personas que por sus condiciones de pobreza, necesitan recursos para sobrevivir.

Complementa lo expresado, Águila (2014), para quien son muchas y distintas las formas en las cuales la explotación puede desarrollarse, pero quizás la más visible sea la trata de personas con fines de explotación sexual, debido a uno de los ámbitos que comprende, la prostitución.

Venezuela no escapa de esta realidad, debido a la crisis socioeconómica presente en el país, la cual se ha acrecentado en los últimos 5 años, es tierra susceptible para que se incremente esta actividad delictiva, la cual viene afianzándose en zonas específicas del país, donde es más fácil captar a potenciales víctimas. Aun cuando ha suscrito convenimientos internacionales, al igual normativas, con la finalidad de detener y minimizar este delito, adicionalmente, tiene un ordenamiento jurídico conformado por varias leyes que lo regulan, los esfuerzos realizados no son suficientes.

Por ello, según lo ha expuesto la Defensoría del Pueblo de la República Bolivariana de Venezuela (2016), especificar de manera rigurosa el tema de la trata de personas en el país, al igual prescribir con exactitud los aspectos que lo caracterizan, resulta un tanto complejo debido a la ausencia de un registro centralizado de casos por parte del ente rector que sistematice las cifras administrativas y judiciales, facilite analizar los perfiles, además georreferencie el delito, visualice sus rutas, así como las consecuencias y causas para determinar los efectos. (p.21).

En función de este señalamiento, la investigación tiene como propósito analizar la trata de personas para el aprovechamiento sexual en Venezuela. 


\section{Revista científica, arbitrada e indizada, bajo la modalidad electrónica.}

\section{REFERENCIAL TEÓRICO}

\section{Trata de personas}

La Trata de personas constituye uno de los principales problemas globales que de acuerdo a la Organización de las Naciones Unidas (ONU) y de la OIT, es uno de los delitos más lucrativos en el mundo, luego del narcotráfico y tráfico de armas.

Para el año 2000, la lucha contra la trata de seres humanos comenzó a formar parte de la agenda de las Naciones Unidas, con la firma de la Convención de las Naciones Unidas contra la Delincuencia Organizada Transnacional y sus Protocolos, instrumento que persigue prevenir, reprimir y sancionar la trata de personas, especialmente de mujeres y niños, en aras de eliminar ese comercio censurable de seres humanos.

En ese sentido, el Protocolo contra la trata de personas contiene una definición convenida por la comunidad internacional, en el apartado a) del artículo 3 de dicho Protocolo, el cual define trata de personas de la siguiente manera:

Por 'trata de personas' se entenderá la captación, el transporte, el traslado, la acogida o la recepción de personas, recurriendo a la amenaza o al uso de la fuerza u otras formas de coacción, al rapto, al fraude, al engaño, al abuso de poder o de una situación de vulnerabilidad o a la concesión o recepción de pagos o beneficios para obtener el consentimiento de una persona que tenga autoridad sobre otra, con fines de explotación. Esa explotación incluirá, como mínimo, la explotación de la prostitución ajena u otras formas de explotación sexual, los trabajos o servicios forzados, la esclavitud o las prácticas análogas a la esclavitud, la servidumbre o la extracción de órganos.(UNODC, 2004, pp. 44-45).

Otro concepto sobre trata de seres humanos, derivado del anterior, es el concebido por la Oficina de las Naciones Unidas contra la Droga y el Delito (UNODC) que la define como: "El delito de trata de personas consiste en utilizar a una persona con fines de explotación para obtener provecho propio o de un tercero, haciendo uso de la coerción o la limitación de la libertad individual” (UNODC, 2009, p. 28).

Para Villalobos (2020), la Trata de personas es:

Un delito que implica graves violaciones de los Derechos Humanos de las personas, por lo que se constituye un delito de lesa humanidad, que afecta y vulnera la dignidad de las personas que son sometidas a estas prácticas 


\section{Revista científica, arbitrada e indizada, bajo la modalidad electrónica.}

crueles y degradantes, por lo que se le ha denominado la esclavitud del siglo XXI. (p.20).

Con relación a lo planteado, de acuerdo a Chávez et al., (2014), América Latina se destaca por ser una zona de origen, tránsito y destino de trata interna e internacional, en la que "la trata de personas, principalmente de mujeres y niñas o adolescentes, se centra en la explotación sexual” (p.18). Según la UNODC (2018):

En esta subregión, la gran mayoría de las víctimas detectadas de la trata son mujeres, lo que representa más del 80 por ciento por ciento del total en el 2016. Si bien las mujeres representan una la mayoría de las víctimas detectadas (51 por ciento), son también una parte significativa las víctimas infantiles detectadas (37 por ciento). Las niñas se detectan con mucha más frecuencia que los niños.

Los países andinos informan de una participación particularmente elevada en la trata de niños. En el Estado Plurinacional de Bolivia y Perú, se detectaron más víctimas infantiles que adultos. En Ecuador, los niños representan poco menos de la mitad de víctimas de trata detectadas. (p.76).

De igual forma, en Venezuela, país señalado como uno de los Estados en donde incide este tipo de delito, ha venido siendo eco de continuas denuncias por considerarse proclive al tránsito de la trata de personas. Según cifras dadas por diferentes organizaciones: De la sociedad civil, internacionales y datos oficiales de otros países de la región, la magnitud del problema es alarmante y en crecimiento. No obstante, a través de los años, el gobierno viene abordando el delito de trata de personas por medio de la Dirección General de Prevención del Delito del Ministerio del Poder Popular para relaciones interiores, justicia y paz.

Sin embargo, diversos estudios han dejado en evidencia, que la redacción de las leyes y normas en el país, lo que demuestra es incapacidad para abordar de manera integral este terrible delito.

Como ejemplo son señalados los datos proporcionados por el Informe global sobre trata de personas difundido por la UNODC (2018), en el cual puede leerse:

En 2018, un total de $\mathbf{4 3 0}$ víctimas venezolanas de trata de personas se identificaron en 21 países de América del Sur, Centroamérica y Europa". Esa cifra contrasta con los datos de otros años: en 2011 se contabilizaron 


\section{Revista científica, arbitrada e indizada, bajo la modalidad electrónica.}

únicamente 5 víctimas venezolanas en el extranjero. En 2012 fueron 11, y en 2013, solo 2. (p.76).

Chávez et al., (2014), consideran que en Latinoamérica el tema de la trata ha ido incrementando, pues el fenómeno es complejo y dinámico en el que intervienen diferentes factores, pero que afecta cada vez más a los menos favorecidos, producto de las relaciones de mercado y consumo. Al respecto, manifiestan que:

Las personas en situaciones de vulnerabilidad social e insatisfacción de necesidades básicas, como empleo, vivienda, educación, acceso a los servicios de salud, entre otras, en sus intentos de búsquedas de nuevas oportunidades de vida se trasladan a espacios geográficos y localidades donde consideran que existen mayores oportunidades de trabajo y de mejoramiento de calidad de vida. Esta movilidad geográfica de las familias a lo largo y ancho de los países de Latinoamérica propicia condiciones de extrema vulnerabilidad y riesgos sociales donde se producen de manera vertiginosa la explotación humana y las diversas modalidades de trata de personas (p. 34).

De igual manera, Villalobos (2020), considera que justamente ese estado de vulnerabilidad que conlleva a la migración "hace de la trata un fenómeno aprovechado por los tratantes para desarrollar nuevas formas de reclutamiento, sobre personas que viven los efectos del desarraigo, la discriminación y xenofobia en contextos que le son ajenos" ( $p$. 19).

\section{Aprovechamiento sexual}

Al hablar de aprovechamiento, se hace referencia a la obtención de algún tipo de beneficio o uso útil de algo, esta acción está vinculada a una actividad o situación, entre otras alternativas. Pero el aprovechamiento sexual, para efectos de esta investigación, será entendido como abuso o extralimitación, utilizada por una persona o grupo delictivo sobre otra, que estando en estado de vulnerabilidad, indefensión o subordinación, se le impone condiciones de diversas acciones sexuales no consentidas por la misma. (El autor, 2021).

En este sentido, el aprovechamiento sexual se puede considerar una de las formas de la violencia sexual contra la mujer, en la que el agresor se impone frente al estado vulnerable de la mujer. 


\section{Revista científica, arbitrada e indizada, bajo la modalidad electrónica.}

La violencia sexual, incluye la explotación y abuso; y se refiere a cualquier acto, intento o amenaza de naturaleza sexual que resulta, o es probable que resulte, en daño físico, psicológico y emocional (ACNUR, 2006, p. 10).

Para Mesa (2020), contrario a la opinión común:

La trata de mujeres con fines de aprovechamiento sexual, no es un crimen que sucede simplemente a una persona de manera aislada, en un momento dado. Este es un hecho más complejo que está socialmente condicionado y configurado desde las lógicas de poder desiguales para hombres y mujeres, abonado por la vulnerabilidad a la que ellas están sujetas comúnmente y exacerbado por la situación país a la que han sido sometidas las venezolanas y su subsecuente deseo/necesidad de emigrar. (p.87).

A lo destacado por Mesa (2020), es necesario agregar algunos aspectos señalado en el informe expuesto por InSight Crime (2021), en el cual expresan:

Un reporte sobre la trata de personas en el estado Bolívar en Venezuela muestra que los campos de trabajo forzado y las redes de tráfico sexual parecen crecer en las minas ilegales de esa región.

De acuerdo con el informe publicado por el Centro de Derechos Humanos de la Universidad Católica Andrés Bello (UCAB), hombres, mujeres y niños están siendo traficados en el estado Bolívar, en una zona que pertenece a la región minera conocida como el Arco Minero del Orinoco.

Venezolanos desesperados que vienen a esta región en busca de trabajo son forzados a trabajar en las minas bajo condiciones similares a la esclavitud, según los autores del informe. A esto se suma que las mujeres se enfrentan a la explotación sexual...

La mayoría de víctimas de las redes de trata son mujeres (74\%), de las cuales el $25 \%$ son niñas y adolescentes. Sin embargo, debido al alarmante subregistro, las cifras podrían ser más altas.

En este orden de ideas, es frecuente leer en los medios de comunicación, noticias

como la siguiente: "Fue desmantelada una red de tráfico de personas con fines de explotación sexual que tiene ramificaciones en los estados Delta Amacuro (este), Lara (noroeste) y La Guaira (norte) conectados con la República de Trinidad y Tobago".

Venezuela reforzará la vigilancia y adelantará planes sociales en Güiria, desde donde se accede a una ruta que está "tomada por mafias que se dedican al tráfico ilegal de emigrantes e incluso a la trata de personas". 


\section{Revista científica, arbitrada e indizada, bajo la modalidad electrónica.}

No obstante, ubicar estadísticas se hace bastante difícil, porque por el tipo de delito a veces resulta imposible concretar la cantidad de casos que realmente existen.

Por otro lado, profundizando en lo planteado, según la Organización Observatorio de mujeres. ONG. (2020), en informe presentado reseña:

La zona andina venezolana, conformada por los Estados Táchira, Mérida y Trujillo, debido a su cercanía con la frontera colombiana, sufre un impacto específico y diferenciado en el contexto de la crisis humanitaria venezolana, en el que observamos un grave deterioro de la calidad de vida de las mujeres, quienes han visto mermados sus salarios y oportunidades laborales, lo que las ha obligado a emigrar. Desde 2017, las mujeres migrantes por población pendular (que cruzan las fronteras frecuentemente) o porque abandonan el país, se encuentran en constante riesgo, lo que ha configurado patrones de trata y tráfico de personas, prostitución forzada, violaciones, embarazos no deseados, engaños con ofertas de trabajo de altos ingresos en divisas o traslados hacia la ciudad de destino con precios económicos; estando más expuestas las adolescentes desde los 14 años hasta las adultas de 25 años. (p.1).

De igual forma, señalan algunas consideraciones que deben ser conocidas por los organismos e instituciones encargadas de monitorear este tipo de actividades:

En el Conversatorio virtual Mujeres Migrantes en las regiones de la frontera colombo venezolana, en el que participaron expertos sobre el tema en el contexto de las zonas de La Guajira y Arauca; se presentó el Boletín Informativo "Afectaciones diferenciales en el marco de la emergencia sanitaria por la COVID-19" publicado por el Observatorio de Asuntos de Género del Norte de Santander (2), en el que plantean como parte de la problemática, la sexualización de los cuerpos de las mujeres migrantes, pues aunque no existen cifras, algunas mujeres entrevistadas migrantes y refugiadas, manifestaron dedicarse a la prostitución como resultado de la coacción por redes de trata de personas con fines de explotación sexual, tanto en el departamento del Norte de Santander, como en el de Arauca y La Guajira; por no conseguir un status migratorio legal, puesto que salen de territorio venezolano sin pasaporte, debido a los múltiples trámites y el alto costo que resulta inaccesible para las mujeres de menos recursos. (p.2).

\section{ORIENTACIÓN METODOLÓGICA}

La investigación de acuerdo a su naturaleza, se encuentra inmersa en un enfoque postpositivista, siguiendo los lineamientos del paradigma cualitativo, de igual manera, es de tipo documental sociojurídico, con un diseño bibliográfico. Enfatizando su preminencia con 


\section{Revista científica, arbitrada e indizada, bajo la modalidad electrónica.}

aportes de un paradigma interpretativo y multi-métodos. Fundamentando estos criterios en Pelekais et al., (2015) y Pelekais et al., (2016).

En síntesis, el trabajo desarrollado, guía su accionar en el diseño bibliográfico, ya que la premisa fundamental es la investigación documental, utilizando para ello como fuentes de consulta, libros, manuales, bases de datos, revistas de investigación científica, repositorios institucionales, libros, leyes, reglamentos, normativas, protocolos, convenios y acuerdos internacionales.

De igual forma, se sitúa en el paradigma interpretativo, el cual tiene como propósito percibir e interpretar la realidad con un carácter comprensivo, implicando el conocimiento de nuevas direcciones, además de extraer conclusiones en horizontes de comprensión más amplios, debido a que persigue: Describir el hecho en el cual se desarrolla el acontecimiento, donde el uso del paradigma cualitativo, consiente hacer una rigurosa descripción contextual de estas situaciones posibilitando la intersubjetividad en la captación de la realidad, por medio de una recogida sistemática de los datos que admite el análisis descriptivo. Apostando por la pluralidad de métodos y la utilización de estrategias de investigación específicas, propias de la condición humana (Pérez Serrano, 1994, p.17), citado por Pelekais et al., (2015).

El universo documental se encuentra conformado por 47 documentos. El trabajo desarrollado da inicio con la elección del tema, realizando un acopio bibliográfico, seguidamente, los datos recopilados de los referentes teóricos son registrados en fichas hemerográficas y bibliográficas, para tenerlos disponibles y localizarlos de manera oportuna. Luego son sistematizados todos los datos, a los fines de efectuar el análisis de contenido.

\section{ANÁLISIS E INTERPRETACIÓN DE LOS RESULTADOS}

En primer lugar se evidencia que Venezuela está muy lejos de erradicar esta actividad delictiva. Son muchos los factores que inciden, tanto externos como internos, sin embargo según el gobierno nacional están siendo implementadas medidas e incluso un plan nacional contra la trata 2020-2025, el cual no fue posible ubicar, para poder conocer las estrategias y acciones que se encuentran formuladas. 


\section{Revista científica, arbitrada e indizada, bajo la modalidad electrónica.}

De igual forma queda queda demostrado que las causas de la trata de personas en el transcurrir del tiempo, independientemente del contexto geográfico con el cual se encuentra relacionado este tipo de actividad delictiva, no ha variado, continúan mencionándose las dificultades económicas, porque precisamente las víctimas de escasos recursos, tienen mayores motivaciones para migrar en búsqueda de mejores oportunidades de vida, por lo tanto resulta más fácil engañarlas, ofreciendo incentivos que a la final no son cumplidos.

Es evidente que aun cuando existe un amplio marco legal que regula la trata de personas en el país, el avance de esta actividad delictiva no se detiene, por el contrario va asumiendo nuevas modalidades de acuerdo a las situaciones contextuales que se generan, tal es el caso del COVID-19. En este sentido, es necesaria una legislación actualizada de acuerdo a las exigencias propias del caso.

El análisis de las fuentes de información ha dejado en evidencia, que en Venezuela son implementados todos los tipos de aprovechamiento sexual descritos, enfatizando en cada uno de ellos, de acuerdo a la región.

Del análisis e interpretación de los resultados, se evidencia que Venezuela está muy lejos de erradicar esta actividad delictiva, la cual azota a todos los países, sin distingo alguno. Pudo corroborarse que en el país hay estados donde se observa una mayor frecuencia del delito. Tales como: Nueva Esparta, Anzoátegui, Apure, Sucre, Aragua, Falcón, Vargas, Carabobo, el Área Metropolitana de Caracas, Bolívar, Táchira y Zulia. Con otro agravante, a partir del 2019, producto del alto porcentaje de migraciones, esta actividad delictiva se ha incrementado en las poblaciones fronterizas.

Por otro lado, tal como es mencionado en el análisis e interpretación de resultados, son muchos los factores que inciden, tanto externos como internos, sin embargo, según el gobierno nacional están siendo implementadas medidas e incluso un plan nacional contra la trata 2020-2025, el cual no fue posible ubicar, para poder conocer las estrategias y acciones que se encuentran formuladas.

\section{CONCLUSIONES}

Ha quedado plenamente demostrado, que las causas de la trata de personas en el acontecer del tiempo, independientemente del contexto geográfico con el cual se encuentra 


\section{Revista científica, arbitrada e indizada, bajo la modalidad electrónica.}

relacionado este tipo de actividad delictiva, no ha variado, continúan mencionándose las dificultades económicas y la insatisfacción de necesidades, de manera prioritaria, porque precisamente las víctimas de escasos recursos son las más vulnerables, tienen mayores motivaciones para migrar en búsqueda de mejores oportunidades de vida, sin importarles la forma de hacerlo, por lo tanto resulta más fácil engañarlas, ofreciéndoles incentivos, que a la final no son cumplidos.

Es notorio de acuerdo con la información revisada, que los traficantes han incorporado la tecnología a su actividad delictiva. En los últimos 4 años, el uso de las redes sociales por parte de los tratantes para la captación de víctimas, es usual. Y como estrategia, los delincuentes suelen enamorar a sus potenciales víctimas, ofreciendo una mejor vida. Se trata de una manipulación, para ganarse su estima y confianza.

Los resultados emanados del análisis efectuado, dejan en evidencia que aun cuando no existe una ley que regule específicamente este delito, si hay un amplio marco jurídico que puede ser utilizado para hacer frente a esta actividad delictiva, tal es el caso de la Constitución de la República Bolivariana de Venezuela, el Código Penal, la Ley Orgánica sobre el Derecho de las Mujeres a una Vida Libre de Violencia, la Ley Orgánica contra la delincuencia organizada y financiamiento al terrorismo, la Ley Orgánica para la Protección del Niño, Niña y Adolescente (LOPNNA).

Los resultados arrojados al develar la información documental examinada, evidencian que los más conocidos de acuerdo a las estadísticas llevadas por los organismos internacionales son: Abuso sexual, explotación sexual, acoso sexual, prostitución forzada, prostitución 2.0, violación, hostigamiento, matrimonio forzoso y trata o esclavitud sexual (siendo este último, uno de los que han sido identificados con mayor presencia desde el 2019, en los estados Bolívar y Apure).

Cada uno de estos tipos de aprovechamiento sexual, tiene unas características muy particulares y en el caso de Venezuela, están presentes en diferentes contextos geográficos del país, aunque en algunos estados es posible encontrar dos o tres tipos diferentes al mismo tiempo. Por ejemplo, las zonas del país donde se concentran mayor número de casos de explotación sexual son en los estados Nueva Esparta, Anzoátegui, Sucre, Aragua, Falcón, Vargas, Carabobo, el Área Metropolitana de Caracas, Bolívar y Zulia. 


\section{Revista científica, arbitrada e indizada, bajo la modalidad electrónica.}

En las zonas fronterizas del Táchira, han sido denunciados con mayor incidencia, los casos de abuso sexual y prostitución forzada. Encontrándose casos de menores de edad, que son vendidos por sus padres para que tengan sexo por hora, como forma de conseguir recursos económicos para comer.

Resulta evidente que la situación socioeconómica ha llevado a una cantidad bastante considerable de personas, a salir del país por diferentes vías y a diversos países, este hecho apalanca el fortalecimiento de la actividad delictiva, porque los traficantes aprovechan ese escenario para penetrar con sus estrategias manipuladoras, aquellos sectores con mayor vulnerabilidad.

Es indudable que el aprovechamiento sexual, producto de la trata de personas se ha infiltrado en la sociedad venezolana, a todos los niveles, deteriorando más aun la calidad de vida de la población.

\section{RECOMENDACIONES}

Invertir en políticas públicas, que atiendan la fractura del tejido familiar, de igual forma, al mejoramiento de las condiciones socioeconómicas de la población del país, a los fines de ir eliminando o por lo menos minimizando este grave flagelo que crece a pasos agigantados, penetrando cada día más sectores, especialmente aquellos con mayor vulnerabilidad.

Considerando que uno de los elementos que ha venido alimentando el problema de la trata de personas es la migración, producto de la extrema pobreza, inseguridad, deficiencia en los servicios públicos, así como la falta de capacidad del motor gubernamental para satisfacer las necesidades de la población. Se recomienda, formular estrategias encaminadas a erradicar estos factores, reactivando al sector empresarial para que haya mayor oportunidad de empleo y generación de ingresos, estabilizar los precios de los productos alimentarios, minimizar la inflación, apuntalar la actividad bancaria y comercial, recuperar el sistema de salud, propulsar un sistema educativo de calidad.

Alinear el ordenamiento jurídico nacional con el internacional en materia de trata de personas y aprovechamiento sexual, a los fines de contar con mayor seguridad jurídica, para hacerle frente a este flagelo azotador. El objetivo es contar con un marco normativo 


\section{Revista científica, arbitrada e indizada, bajo la modalidad electrónica.}

que facilite la lucha contra esa actividad delictiva, de manera que pueda garantizarse un proceso judicial efectivo, para condenar a los perpetradores del hecho.

Brindar protección y asistencia efectiva a las víctimas de esta actividad delictiva, proporcionando los recursos necesarios para su recuperación física, psicológica y social.

\section{REFERENCIAS BIBLIOGRÁFICAS}

ACNUR (2006). Directrices sobre protección internacional: La aplicación del artículo 1A(2) de la Convención de 1951 o del Protocolo de 1967 sobre el Estatuto de los Refugiados en relación con las víctimas de la trata de personas y las personas que están en riesgo de ser víctimas de la trata. [Documento en línea]. Disponible: https://bit.ly/3szcFv2. [Consulta: 2020, Noviembre 15].

Águila, M. (2014). La trata de seres humanos con fines de explotación sexual. Análisis comparativo del marco jurídico internacional, nacional y local. Revista Internacional de Pensamiento Político - I Época - Vol. 9 - 2014 - [399-423] - ISSN 1885-589X.

Asamblea Nacional de la República Bolivariana de Venezuela. Ley reforma parcial del Código Penal. (2005). Gaceta Oficial 5763 E del 03 de marzo de 2005. Disponible en: https://es.slideshare.net/heglesaudade/codigo-penal-45685581

Asamblea Nacional de la República Bolivariana de Venezuela. Ley Orgánica contra la Delincuencia Organizada y Financiamiento al Terrorismo. Gaceta Oficial $\mathrm{N}^{\circ}$ 39.912. 30 de abril de 2012. Caracas. Venezuela.

Chávez, M., Ramírez, E., Beracoechea, A (2014). La Trata y el tráfico de personas: Escenarios globales de la esclavitud postmoderna. [Revista en línea].Disponible:http://www.ixaya.cucsh.udg.mx/sites/default/files/1_la_trata_y_el trafico_de_personas.pdf. [Consulta: 2020, Noviembre 12].

Defensoría del Pueblo de la República Bolivariana de Venezuela. (2016). Diagnóstico sobre las Políticas Públicas y la Respuesta Institucional de los Estados Contra la Trata de Personas en la Región Andina. Disponible en: http://repositorio.dpe.gob.ec/bitstream/39000/1311/3/CT-007-2016.pdf

InSight Crime (2021). La trata de personas, otro mal del arco minero de Venezuela. Disponible en: https://es.insightcrime.org/noticias/trata-personas-arco-minerovenezuela.

Ley Orgánica para la Protección al Niños, Niñas y Adolescentes, publicada en Gaceta Oficial Extraordinaria No 6185, de fecha 8 de junio 2015.

Ley Orgánica sobre el derecho de las mujeres a una vida libre de violencia (2014). Gaceta Oficial No. 40.548 de fecha 25 de noviembre de 2014. 


\section{Revista científica, arbitrada e indizada, bajo la modalidad electrónica.}

Mesa, V. (2020). La trata con fines de explotación sexual: El más reciente peligro para las venezolanas. [Revista en línea]. Disponible: $\underline{\text { https://dialnet.unirioja.es/servlet/articulo?codigo=7574152 [Consulta: 2021, Marzo }}$ 10].

Oficina de Las Naciones Unidas Contra la Droga y el Delito (UNODC) (2004). Convención de las naciones unidas contra la delincuencia organizada transnacional y sus protocolos. [Documento en línea]. Disponible: https://www.unodc.org/documents/treaties/UNTOC/Publications/TOC\%20Conventi on/TOCebook-s.pdf. [Consulta: 2020, Noviembre 15].

OMS (2013). Comprender y aboradar la violencia contra las mujeres. USA. Disponible en: https://oig.cepal.org/sites/default/files/20184_trata.pdf

Organización Observatorio de mujeres, ONG. (2020). Informe sobre la trata de mujeres y explotación de la prostitución en la frontera colombo-venezolana Disponible en: http://observatorioddhhmujeres.org/informes/trata $\% 20 \mathrm{de} \% 20$ mujeres $\% 20 \mathrm{y} \% 20 \mathrm{expl}$ otaci\%C3\%B3n\%20de\%20la\%20prostituci\%C3\%B3n.pdf

Pelekais, C., Pertuz, F., Pelekais, E. (2016). Hacia una cultura de investigación cualitativa. Ediciones Astro Data S.A. Maracaibo. Venezuela.

Pelekais, C; El Kadi, O; Seijo, Cristina; Neuman, N. (2015). El ABC de la Investigación. Pauta Pedagógica. Ediciones Astro Data S.A. Maracaibo. Venezuela.

Rivero, R. (2021. Trata de personas para el aprovechamiento sexual en Venezuela. Trabajo de Grado Universidad Yacambú. Venezuela.

UNODC (2009). Manual sobre la investigación del delito de trata de personas. Guía de autoaprendizaje. [Documento en línea]. Disponible: https://www.unodc.org/documents/human-trafficking/AUTO_APRENDIZAJE.pdf. [Consulta: 2020, Noviembre 15].

UNODC (2018). Informe Global de Trata de Personas 2018, con énfasis en Sudamérica. Disponible en: https://www.unodc.org/bolivia/es/La-UNODC-presento-el-InformeGlobal-de-Trata-de-Personas-2018-con-enfasis-en-sudamerica.html

Villalobos, N. (2020) Protección de las víctimas de trata de personas y similitudes con la protección de las personas en necesidad de protección internacional. [Revista en línea]. Disponible: https://dialnet.unirioja.es/servlet/articulo?codigo=7574152 [Consulta: 2020, Noviembre 12]. 